\title{
La perversión de la educación imperialista
}

Dr. Agustín Velloso Santisteban

Doctor en Ciencias de la Educación

Universidad Nacional de Educación a Distancia (UNED), España

avelloso@edu.uned.es

Fecha de recepción: 29 de noviembre, 2019 / Fecha de aceptación: 03 de diciembre, 2019

https://doi.org/10.5377/torreon.v8i22.9029

Palabras clave: imperialismo, sistema educativo nacional, educación militarista

\section{RESUMEN}

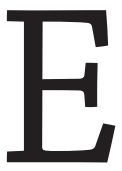

1 imperialismo sigue vivo hoy como hace siglos, aunque con mayor fuerza. Esto se manifiesta en el aumento de su control sobre las poblaciones sometidas y su ingente acaparamiento de los recursos mundiales. Para mantener esta posición emplea, también hogaño como antaño, la cañonera, la religión y la educación. Dentro de la educación formal, mayoritariamente estatal, los gobiernos emplean el sistema educativo como una herramienta más para promover el imperialismo. Su éxito principal es haber logrado que las poblaciones sometidas se resignen a su destino.

\section{INTRODUCCIÓN}

Hace más de 30 años que el economista Martin Carnoy publicó su libro titulado Education as Cultural Imperialism Carnoy (1987), en el que escribió:

"la educación formal de Occidente llegó a muchos países como parte de la dominación imperialista."

Añadió que esto "era consecuente con los fines del imperialismo: el control económico y político de la gente de un país por la clase dominante de otro. El poder imperial intentaba, por medio de la educación escolar, preparar a los colonizados para las funciones que convenían al colonizador". Carnoy (1987, p. 13-14). 
Esta situación no ha variado en lo esencial: ni en cuanto al control citado ni en lo que se refiere a la forma de extender el imperialismo por doquier. Nadie puede llevarse a engaño. Más bien todo el mundo lo sabe aunque sólo unos pocos se atreven a decir la verdad en público y con ello ponerse en peligro.

Esto es lo que hizo el coronel Muamar al Gadafi en la sede de la Organización de las Naciones Unidas (ONU) en su 64ª Asamblea General, en septiembre del año 2009 y que aquí conviene recordar. Dijo refiriéndose a la Carta de las Naciones Unidas (de forma muy extractada):

"El Preámbulo es muy atractivo, y nadie lo objeta, pero todas las disposiciones que aparecen después lo contradicen completamente (...). Se dice que tenemos igualdad de derechos, ya sean nuestros países grandes o pequeños. ¿Tenemos nosotros el derecho de veto? ¿Somos iguales? El veto contradice la Carta. (...). En el Preámbulo de la Carta se afirma que no se deberá recurrir a la fuerza armada, salvo en interés común pero ¿qué ha sucedido desde entonces? Han estallado 65 guerras desde la creación de las Naciones Unidas y del Consejo de Seguridad, con millones de víctimas más que en la segunda guerra mundial (...) ¿Acaso esas guerras, así como la agresión y la fuerza que se utilizaron responden al interés común de todos nosotros? Eso contradice de manera flagrante la Carta de las Naciones Unidas que firmamos (...). Conceder el derecho de veto de los miembros permanentes del Consejo de Seguridad a los que tienen el poder es una injusticia y un acto terrorista, y no deberíamos tolerarlo. No deberíamos vivir a la sombra de esta injusticia y del terror. Las superpotencias tienen intereses mundiales complejos y usan el derecho de veto para proteger esos intereses." (Ramos, 2016), (Lèvesque, 2013).

Lamentablemente no se han cumplido todavía sus palabras "Estamos a punto de enjuiciar a las Naciones Unidas; la antigua Organización llegará a su fin y surgirá una nueva", la ONU sigue corrompiéndose; sigue violando la letra y el espíritu de la Carta. Puede señalarse que la paz mundial y el resto de sus objetivos declarados están más lejos que nunca.

\section{DESARROLLO}

Los medios del imperialismo evolucionan merced a las nuevas tecnologías y otros recursos a su disposición. El fin no varía como no sea el de aumentar más su dominio sobre la tierra y paulatinamente también del espacio exterior.

¿Setrata solamente de un avance cuantitativo? Desdeluego que elimpulso delimperialismo se incrementa, aunque también se puede hablar de un avance cualitativo en paralelo.

Antiguamente, era la fuerza de las cañoneras la que imponía la voluntad del imperialista sobre las 'razas inferiores'. La educación y la religión contribuían a que estas se resignaran de alguna manera a su destino y evitaran ser el objetivo de los cañonazos.

La educación imperialista estaba destinada a proporcionar a las poblaciones sometidas los conocimientos que les permitían trabajar en actividades favorables al imperio y como 
'efecto colateral' (en el lenguaje imperialista de hoy) ganarse la vida, aunque esto es meramente instrumental, es decir, en la medida en que contribuía al primer objetivo.

A partir de la Segunda Guerra Mundial se produce el fenómeno de la descolonización, que para resumir en dos palabras se puede afirmar que su resultado ha enriquecido a las metrópolis y empobrecido a las colonias, (Descolonización, 2019).

Esto es así porque debido al coste pagado en el pasado por las naciones colonizadas y expoliadas en vidas humanas y bienes, estas aún no se han repuesto, (Naciones Unidas y la Descolonización, 2015).

Tanto ha sido el éxito del imperialismo que no solamente no se ha producido su fin, sino que evoluciona a toda marcha y de esta forma lo mismo el daño y el perjuicio.

Es recomendable detenerse en el título de la citada "Declaración sobre la concesión de la independencia a los países y pueblos coloniales" y en el breve texto previo a la declaración propiamente dicha.

El primer párrafo de la Declaración reconoce que "La sujeción de los pueblos a una subyugación, dominación y explotación extranjeras constituye una denegación de los derechos humanos fundamentales, es contraria a la Carta de las Naciones Unidas y compromete la causa de la paz y de la cooperación mundiales".

El conjunto es un alambique tan absurdo y fingido que muestra la enorme disparidad de fuerzas entre los dos bandos y anticipa lo que faltaba por llegar.

Lo que ocurrió en realidad en la etapa posterior a la Declaración fue un simple cambio de nombre: de colonialismo a neocolonialismo, con el que se entendía que los países recién liberados lo eran políticamente pero no económicamente.

Ese desabrido término dio lugar a otro, el cual sigue presente en la actualidad: 'globalización'. Quizás algún día se escribirá y se estudiará que esto no es más que el envoltorio de un caramelo envenenado, pues la descripción de sus resultados se ajusta perfectamente al primer párrafo de la Declaración 1514.

El colonialismo, aunque levemente, había recibido una reprimenda en la sede de la ONU por parte de la mayoría de sus países miembros, pero aquella minoría colonialista tenía una respuesta fácil: cambiar el nombre y dejar que la realidad siguiera su curso, es decir, permaneciese bajo su control.

Esta perversión del lenguaje se usa desde entonces ad nauseam. En el Foro Boao de Asia, celebrado en abril de 2018, el Secretario General de la ONU, António Guterres, afirmó que “estoy profundamente convencido de que la globalización es irreversible (...) ha traído muchos 
beneficios", (At Asian forum, UN chief calls for more equitable globalization, urgent action on climate change, 2018).

Es cierto que añadió: "El mundo debe actuar para extender los beneficios de la globalización de forma más equitativa" (At Asian forum, UN chief calls for more equitable globalization, urgent action on climate change, 2018), lo cual es una falsedad, ya que la riqueza producida, mucha o poca, no se distribuye de forma equitativa como lo muestra la distancia creciente entre los que más poseen y los que menos.

La mejora continua de la propaganda, la publicidad y los medios de comunicación de masas fue y es de mucha utilidad. Esto tiene interés recordarlo antes de pasar al papel de la educación, porque es razonable argumentar que el mayor éxito de la globalización no es haber conseguido que los ricos sean más ricos y que los pobres sean más pobres, sino que gracias a esos medios y sus mensajes que les narcotizan y alienan, estos se encuentren resignados.

Obviamente, es discutible el número de los que se resignan, los que la aceptan con mejor o peor disposición y cuántos activamente resisten. Lo que es menos discutible es el cruel sufrimiento programado que ocasiona a millones sin cuento de seres humanos y el dañino impacto que causa al planeta, conocido desde hace años sin que se haya tomado las medidas necesarias para detenerlo hasta ahora.

Esto es un sinsentido cuando el estado de la ciencia y la técnica es el más extraordinario que ha conocido la humanidad:

Hoy se puede disparar un misil balístico intercontinental (ICBM por sus siglas en inglés) con diez cabezas termonucleares para que en poco más de media hora impacte en un objetivo que está a 15.000 kilómetros de la base de lanzamiento, pero no hay un artefacto capaz de llevar medicinas y alimentos a poblaciones necesitadas, incluso aunque se estén muriendo días o meses después de hacerse pública su situación.

Esto no parece muy acorde con la Carta de las Naciones Unidas y menos con el espíritu humanitario más básico, ni siquiera con el sentido común.

Además de la labor de los agentes de propaganda (educación no formal) con las masas, las instituciones escolares (educación institucional) tienen un papel no menos importante en la propagación y aceptación de la globalización entre los hijos de esas multitudes.

Desde hace unos años es habitual encontrar entre las enseñanzas tradicionales dedicadas a la formación 'nacional', para la 'democracia' y la 'ciudadanía', que reciben los escolares a lo largo de su permanencia en el sistema educativo obligatorio y más allá, otras nuevas dedicadas a la globalización, las 'intervenciones humanitarias', etc. y también sobre el 'emprendimiento' y la 'educación financiera'. 
Unos ejemplos dan cuenta de lo lejos que ha llegado el imperialismo en el uso de los sistemas educativos para su propio provecho.

El sistema educativo en Palestina es un caso extremo por varias razones conocidas: ocupada militarmente por Israel, sufre la violación permanente de las leyes internacionales por parte de éste, que cuenta con el apoyo de la 'comunidad internacional', (Velloso, 2002, p. 145154).

Esto se traduce en que el sistema educativo sufre las consecuencias como el resto de sistemas nacionales: sanidad, economía, infraestructuras, etc., de las agresiones continuas por parte de Israel que se intercalan entre guerra y guerra: Plomo Fundido (2008-09) Pilar Defensivo (2012) y Frontera Protegida (2014).

Igual que estas guerras contra la población civil palestina se presentan descaradamente ante la opinión pública mundial como 'defensivas', 'operaciones antiterroristas', etc., otras agresiones más reducidas pero constantes se presentan así: "la Universidad de Tulkarem, colocada junto a una instalación militar activa, ha seguido siendo el escenario de choques entre los estudiantes y el personal de las Fuerzas de Defensa de Israel." (FDI), (Bureau of Democracy, Human Rights, and Labor - U.S. Department of, 2016).

Conviene prestar atención a este pervertido lenguaje: la responsabilidad de lo que ocurre se coloca en la universidad palestina y no en las FDI porque según la fuente que informa:

a. está "colocada" junto a una instalación militar activa, cuando es esta la que ha ido a establecerse en Tulkarem, población de Cisjordania, bajo control de Israel como el resto de Palestina;

b. además se presenta como el agente activo: "sigue siendo el escenario" en lugar de "sigue siendo objetivo de los ataques de las FDI", como si fuera la que dispara y no la que es agredida;

c. escribir "choques" entre los estudiantes y el "personal de las FDI" es una mentira superlativa porque los estudiantes -que son civiles desarmados y protegidos por la ley internacional- son las víctimas de los proyectiles que disparan los soldados contra la universidad. Los soldados aparecen como unos simples trabajadores más de la universidad, como un personal habitual en todas las universidades del mundo. Puede hablarse de choques cuando hay proporcionalidad entre los contendientes, pero esto no se corresponde con la realidad: estudiantes indefensos son agredidos por soldados dotados de armas de guerra.

Alguien dirá que existe un proceso de paz entre Israel y Palestina. Cierto, un proceso de que se inició en Madrid en 1991 y de forma oficial desde los Acuerdos de Washington firmados 
entre las partes en 1993 en Washington, pero dura ya más de 25 años según la segunda cifra y es más cierto que la ocupación militar israelí sobre Palestina no se ha terminado, se ha afianzado más y más.

La principal característica del proceso de paz es su incertidumbre. Es una incertidumbre inherente, no centrada únicamente en la velocidad del proceso o en factores coyunturales. Se trata de que las dos partes en el conflicto tienen objetivos contrarios y trabajan en direcciones opuestas. Además, los palestinos son la parte más débil con una diferencia abismal y dependen casi por completo de Israel.

Finalmente, la ley internacional de aplicación al conflicto, aunque no ha sido oficialmente abrogada ha pasado a ser irrelevante en la solución del mismo. La última medida del gobierno de Estados Unidos, anunciada por su Secretario de Estado ha sido declarar que "el establecimiento de asentamientos civiles israelíes en Cisjordania, por sí mismo, no contradice la ley internacional", (BBC, 2019).

Las autoridades educativas palestinas, los profesores, las familias y los ciudadanos en general no pueden evitar enfrentarse a este ambiente esquizofrénico. La incertidumbre respecto de la situación política en el porvenir no ayuda en nada a la clarificación de los objetivos educativos ni a la gestión de los planes de enseñanza.

El problema no está en el sistema educativo: sin el control sobre el Estado no hay control sobre la educación.

Aunque la Autoridad Palestina (AP) mediante su Ministerio de Educación, tiene control nominativo sobre el sistema educativo palestino, carece de control sobre el destino de la nación palestina. A causa de ello, a pesar de que el currículum está pensado para contribuir al logro de los objetivos nacionales, lo cierto es que apenas puede hacerlo.

La pregunta más sencilla de responder para otro estudiante en cualquier otro país del mundo es una tarea ímproba para un niño palestino: ¿cuáles son las fronteras y la extensión de tu país? Mejor no mencionar otras: ¿cuáles son las atribuciones del Estado? ¿Cuál es el destino de los millones de refugiados a quienes no se permite volver a sus casas? ¿Por qué los palestinos no tienen un Estado como lo tienen los israelíes? ¿Cómo han de contribuir los estudiantes a la construcción nacional y al restablecimiento de sus derechos nacionales?

El gobierno palestino no tiene capacidad alguna para solucionar esta situación típicamente colonialista, así que la única salida que le queda es distraer la atención de escolares, profesores y familias, tarea que delegan en las organizaciones no gubernamentales (ONGs) del imperio, (one. laptop.org, 2010). 
Según la página electrónica de 'one laptop per child' (OLPC), "las autoridades educativas palestinas, la UNRWA (la agencia de la ONU para los refugiados palestinos) y la Palestinian Education Initiative (PEI) han colaborado en la campaña internacional OLPC, un ordenador por niño", (one.laptop.org, 2010).

Así se publicita la campaña: "El cliente dona 400 dólares y recibe dos ordenadores portátiles, uno de los cuales va para un niño en un país en desarrollo”, (Shah, 2008).

¿Qué hay detrás de esta generosidad? Una multinacional: www.amazon.com

¿Quién es el dueño de Amazon? Jeff Bezos, el hombre más rico del mundo con 100 mil millones de dólares.

En diciembre de 2016 Bezos visitó Israel para negocios con la Universidad de Haifa y con Israel Aerospace Industries (IAI), (haaretz, 2016). La conclusión es clara: sería ingenuo pensar que el sistema educativo funciona como si estuviese dentro de una burbuja impenetrable por el imperialismo. Muchas de las 'lecciones' que este proporciona a niños y jóvenes giran en torno a la violencia constante y sin fin que incluye asesinatos extrajudiciales y masacres indiscriminadas; que se dirige contra personas, bienes e instituciones, testigos internacionales, contra la ley internacional y contra el porvenir de la población palestina.

¿Cabe esperar que la educación formal sea capaz de enderezar esta situación? Apenas puede, su influencia es mucho menor que la no formal. La enseñanza es que guerras constantes en la zona, muchos más enfrentamientos menores, ataques y contraataques, la ausencia de justicia y una violación generalizada de los derechos humanos, tienen una influencia 'educativa' mucho más poderosa que folletos ministeriales y campañas de organizaciones no gubernamentales.

Por otro lado, también la población de los países imperialistas recibe otra 'educación', aunque adecuada a su situación, bien diferente de la de aquélla.

Hace 120 años el escritor británico Rudyard Kipling escribió para la Reina Victoria con motivo de su jubileo de diamante un poema titulado La carga del hombre blanco con un subtítulo ad hoc: Los Estados Unidos y las Islas Filipinas. En él se alaba la hombría, el esfuerzo, la dignidad, el altruismo y la sabiduría del colonialista occidental, que a su parecer emplea en beneficio de los ingratos salvajes.

Por inmoral y grosero que a un lector contemporáneo le pueda parecer, la situación apenas ha cambiado: hoy ya no es un representante cualificado de la clase imperial el que educa, sino que es el propio Estado y agencias privadas los que emplean instituciones, personal y recursos públicos y privados para el mismo objetivo. 
La Federación de Científicos Americanos (FAS por sus siglas en inglés) fundada en 1945 “tiene la convicción de que científicos, ingenieros y otras personas con formación técnica tienen la obligación moral de asegurarse de que los frutos tecnológicos de su intelecto y esfuerzo se apliquen al beneficio de la humanidad", (fas, 2019).

Uno de los estudios de la FAS informa de que "el Programa de Capacitación del Cuerpo de Oficiales Jóvenes de Reserva (JROTC por sus siglas en inglés) (usarmyjrotc, 2019) es una instrucción voluntaria para estudiantes de segunda etapa de secundaria ofrecida por el Departamento de Defensa (DoD) a través del ejército. Su objetivo es inculcar en los estudiantes el valor de la ciudadanía, el servicio a los Estados Unidos, la responsabilidad personal y un sentido de realización'."

Añade que hay más 3.000 unidades que operan en cada estado y en institutos de ultramar del DoD. En el programa hay más de 500.000 estudiantes matriculados". "La financiación del programa para el año fiscal 2019 fue de 386 millones de dólares”, (Kamar; Arriaga, 2019).

Su origen está en la ley firmada por el presidente George W. Bush justamente después de los atentados del 11 de septiembre de 2001: ley No Child Left Behind, Ningún Niño Desatendido (NCLB, 2001, por sus siglas en inglés, reemplazada en 2015 por la ley Every Student Succeeds Act, Todos los Niños Triunfan de 2015):

"Las escuelas que reciben fondos federales deben dar a los reclutadores militares el mismo acceso a los estudiantes que se les da a los representantes de empresas y universidades que buscan trabajadores y estudiantes respectivamente, lo que incluye los nombres de todos los estudiantes de la etapa secundaria”.

Por si esto no fuese suficiente, "un informe del US Army War College (Escuela de Guerra del Ejército de Estados Unidos) aboga por un reclutamiento sin restricciones, ya que 'el acceso por parte del DoD a los estudiantes de secundaria sigue siendo de la máxima importancia para incrementar la tropa, debido a que la inclinación al servicio militar decae de forma importante entre los mayores de 18 años'."

El DoD negocia con empresas especializadas en el tratamiento de datos para que le provean información sobre los estudiantes de forma que aquél pueda seleccionar los más adecuados a sus fines (...), desarrolla videojuegos, cuenta con colaboración de la industria del cine, por supuesto con la de la publicidad, etc.

Todo este despliegue de personal y recursos incluye también a los preescolares, lo que va promoviendo entre las jóvenes generaciones un pensamiento acerca de la guerra y la milicia como algo excitante y genial, sin que se mencione lo relativo al trauma y la muerte para los que está diseñado, (Grey, 2016).18 
Para situar el programa en perspectiva, lo mejor es completar estos datos y cifras oficiales con el punto de vista de un experto, un veterano de Afganistán, porque ofrece una visión muy alejada de los eslóganes, los valores y el orgullo vistos anteriormente.

Rory Fanning fue un comando del Ejército de Estados Unidos que como veterano visita institutos en la actualidad, pero no lo hace para reclutar, sino que lo hace porque en sus charlas en los centros escolares los estudiantes descubren un mundo del que nadie les ha hablado, (Fanning, 2019).

Primero "describe la frustración que sintió con el sistema escolar de Chicago porque en los barrios más pobres de la ciudad se estaban cerrando escuelas a una velocidad récord y sin embargo de alguna manera siempre tenía dinero para completar la financiación del programa JTROC”. (...) Este conocido programa es la gallina de los huevos de oro para los institutos y los desincentiva de mover la barca en aguas agitadas."

El resto es un relato estremecedor de su paso por el ejército y la guerra, primero durante su formación militar, después durante su misión en Afganistán, muy esclarecedor de sus consecuencias:

"Me alisté en los Army Rangers para pagar mis prestamos escolares y poner mi grano de arena e impedir otro ataque terrorista como el del 11 de septiembre (de 2001) (...) Principalmente creo que los mandos me entrenaban para decir sí a sus órdenes. El ejército y el pensamiento crítico no son compatibles". (...)

"El Ejército daba miles de dólares a cualquiera dispuesto a identificar supuestos miembros del Talibán y los soldados allanábamos casas a partir de esa información. Luego comprendí que esa 'información', si es que se le puede llamar así, tenía su fuente en un tipo de desesperación: la pobreza abyecta de un afgano en busca de cualquier trabajo para sostener a su familia, le lleva a señalar a cualquiera a cambio de acceso al profundo pozo de dinero de que dispone el ejército. En un mundo donde hay pocas fábricas y los empleos administrativos escasean, la gente hará lo que sea para sobrevivir, tienen que hacerlo." (...)

"A menudo echábamos abajo la puerta de una casa y nos llevábamos a un afgano no por su relación con el Talibán o al-Qaeda, sino porque un vecino tenía una pendencia con él." (...)

"Mi compañía ocupó la escuela de un pueblo y el comandante suprimió las clases indefinidamente porque aquélla era un excelente puesto de tiro y no había en las zonas rurales de Afganistán un director de escuela capaz de disuadir al ejército más poderoso y tecnológicamente avanzado de la historia de que hiciese su propia voluntad."

"Recuerdo dos hombres en edad militar que pasaban por la escuela que estábamos ocupando. Uno de ellos no mostró un nivel aceptable de deferencia hacia mi primer sargento, 
así que los detuvimos. Encerramos al prepotente sujeto en un cuarto y a su amigo en otro. El primero escuchó un disparo y pensó, tal y como lo habíamos planeado, que acabábamos de matar a su amigo por no responder a lo que le preguntamos y que él sería el próximo."

"Estoy más orgulloso de haber salido del ejército que de cualquier cosa que hice cuando estaba en él. Mis dos misiones en Afganistán hicieron que me diera cuenta de que estaba haciendo menos seguro al mundo. Hoy sabemos que la mayoría del aproximadamente millón de personas que han resultado muertas desde el 11 de septiembre eran civiles inocentes, gente que no tenía nada que ver con eso y ninguna razón para luchar hasta que, como ocurría a menudo, el ejército le provocaba matando o hiriendo a un familiar que casi siempre no era más que un inocente transeúnte."

"Entiendo que el programa es un sustento vital para la guerra permanente de Washington en Medio Oriente y partes de África. Sus interminables conflictos sólo se producen porque los estudiantes siguen matriculándose. Una y otra vez los políticos y las juntas escolares declaran que sus institutos están sin dinero, no hay dinero para libros, salarios de los profesores y sus pensiones, comidas saludables... Sin embargo, en 2015, el gobierno gastó 598 mil millones en el ejército. Imagínese qué sistema educativo tendríamos en este país si los profesores recibiesen el mismo dinero que reciben los contratistas de armamento. (...) Es difícil luchar en eternas guerras de billones de dólares si los chicos no se alistan, (Fanning, 2016).

\section{CONCLUSIONES}

El imperialismo sigue su curso hoy como hace siglos. La diferencia fundamental entre el antiguo y el nuevo es la aplicación de las nuevas tecnologías a las herramientas de siempre: la cañonera, la religión y la educación. Su objetivo no varía: el dominio de los recursos y el control de las poblaciones.

Hoy el imperialismo aparece disimulado bajo el término globalización, cuyo mayor éxito no es enriquecer más a los ricos a costa de aumentar su distancia económica respecto del resto de la humanidad, sino lograr que sus miembros se resignen a este destino.

La educación también sigue colaborando en la consecución de aquellos objetivos, dentro del sistema escolar y fuera también. En aquél se trata de una educación formal, la cual está principal aunque no exclusivamente en manos del Estado. Fuera de la escuela se realiza por otros medios como la propaganda y la publicidad, con el potente concurso de los medios de comunicación de masas y se conoce como educación informal.

En el caso de Israel, que ocupa militarmente desde hace décadas a Palestina, se evidencia que mediante su ingente labor de propaganda sobre un supuesto proceso de paz y la inexistencia de violaciones de la ley internacional por su parte, intenta convencer al mundo de su posición, lo 
que incluye a los propios palestinos, una población tan castigada y debilitada que apenas puede resistir.

El caso de Estados Unidos expone un país que a pesar de su incomparable poder económico y militar, necesita que sus gobiernos intervengan de varias maneras en el sistema educativo para proveer al ejército de carne de cañón, que será usada en el sometimiento de poblaciones y el control de sus recursos en cualquier lugar del globo.

En ambos casos se aprecia que las empresas privadas forman parte de estas intervenciones estatales en una suerte de alianza en contra de votantes y consumidores, que es a lo que se ven reducidos en realidad los que, sin embargo, se creen que son ciudadanos del siglo XXI, a su juicio mucho más humanizados y avanzados que el de los antiguos colonizados.

\section{REFERENCIAS}

Carnoy, Martin (1987) Education as Cultural Imperialism, New York: David McKay Co.

Carnoy, Martin (1987). La educación como Imperialismo Cultural.

Ramon, Ruben (2016). "Las tales Naciones Unidas no existen": Al Gadafi. Recuperado http://kaosenlared.net/las-tales-naciones-unidas-no-existen-al-gadafi/

Lévesque (2013). Más sobre el imperialismo de Estados Unidos, la Ley Internacional y las Naciones Unidas. Recuperado de www. globalresearch.ca/us-imperialism-international-law-and-the-united-nations/5314381

Descolonización (2019). Recuperado de http://es.wikipedia.org/wiki/Descolonizaci\%C3\%B3n\#Descolonizaci\%C3\%B3n_pol\%C3\%ADtica

Las Naciones Unidas y la Descolonización (2015). Recuperado de https://www. un.org/dppa/decolonization/es/history/ international-decades
At Asian forum, UN chief calls for more equitable globalization, urgent action on climate change (2018). Recuperado de http://news.un.org/en/ story/2018/04/1006951

Velloso, Agustín (2002). Palestinian Education: A National Curriculum Against All Odds, International Journal of Educational Development, 22:2, 145-154

Bureau of Democracy, Human Rights, and Labor - U.S. Department of 2016 Country Reports on Human Rights Practices (2016). Recuperado de https://www. state.gov/country-reports-on-human-rights-practices-for-2018/

US says Israeli settlements are no longer illegal (2019). Recuperado de www.bbc.com/ news/world-middle-east-50468025

one.laptop.org (2010). Recuperado de http://one.laptop.org/news/250-palestinian-refugee-children-receive-laptops-amari-boys-school 
one.laptop.org (2010). Recuperado de http:// one.laptop.org/ y http://one.laptop.org/ about/mission

Shah, Agan (2008). Amazon to sell OLPC's XO laptop starting Nov. 17. Recuperado de www.computerworld.com/ article/2534150/mobile-wireless/ amazon-to-sell-olpc-s-xo-laptop-starting-nov--17.html

Haaretz (2016). Recuperado de www.haaretz. com/israel-news/business/1.758632

Fas (2019). Recuperado de http://fas.org/ about-fas/

usarmyjrotc (2019) www.usarmyjrotc.com/ index.php

Kamarc; Arriaga (2019). Defense Primer: Junior Reserve Officers' Training Corps (JROTC). Recuperado de http://fas.org/ sgp/crs/natsec/IF11313.pdf
Grey (2016). Why Is My Kindergartner Being Groomed for the Military at School? Recuperado de http://truthout.org/articles/why-is-my-kindergartner-beinggroomed-for-the-military-at-school/

Fanning (2019). Fighting to End the Unending Wars. Recuperado de http://roryfanning.org/

Fanning (2016). Tomgram: Rory Fanning, Talking to the Young in a World That Will Never Truly Be "Postwar". Recuperado de www.tomdispatch.com/blog/176125/ tomgram\%3A_rory_fanning\%2C_talking_to_the_young_in_a_world_that_ will_never_truly_be_\%22postwar\%22 